\title{
Lorentz forces induce inhomogeneity and flux in active systems
}

\author{
H. D. Vuijk, ${ }^{1}$ J. U. Sommer $\odot,{ }^{1,2}$ H. Merlitz $\odot,{ }^{1}$ J. M. Brader, ${ }^{3}$ and A. Sharma $\oplus^{1,2, *}$ \\ ${ }^{1}$ Leibniz-Institut für Polymerforschung Dresden, Institut Theorie der Polymere, 01069 Dresden, Deutschland \\ ${ }^{2}$ Technische Universität Dresden, Institut für Theoretische Physik, 01069 Dresden, Deutschland \\ ${ }^{3}$ Department de Physique, Université de Fribourg, CH-1700 Fribourg, Suisse
}

(Received 8 August 2019; accepted 23 January 2020; published 16 March 2020)

\begin{abstract}
We consider the dynamics of a charged active Brownian particle in three dimensions subjected to an external magnetic field. We show that, in the presence of a field gradient, a macroscopic flux emerges from a fluxfree system and the density distribution becomes inhomogeneous. The flux is induced by the gradient of the magnetic field only and does not require additional symmetry breaking such as density or potential gradients. This stands in marked contrast to similar phenomena in condensed matter such as the classical Hall effect. We further demonstrate that passive tracer particles can be used to measure the essential effects caused by the Lorentz force on the active particle bath, and we discuss under which conditions this diffusive Hall-like effect might be observed experimentally.
\end{abstract}

DOI: 10.1103/PhysRevResearch.2.013320

\section{INTRODUCTION}

Lorentz force may appear irrelevant in soft-matter systems, which are dominated by overdamped diffusive dynamics. However, it is detectable in, for example, soft tissues where it can be used for imaging applications [1]. In systems with overdamped dynamics, the Lorentz force reduces the diffusivity in the plane perpendicular to the magnetic field, which implies that the Fokker-Planck equation (FPE) requires a tensor. This may appear to be trivial, were it not for the recent finding that the tensor has an antisymmetric part, which gives rise to fluxes in the direction perpendicular to density gradients, thereby precluding a diffusive description of the dynamics $[2,3]$. Although some aspects of the nondiffusive dynamics have been explored in passive systems, little is known about its effect on active systems.

In contrast to externally driven systems, active matter has the hallmark of being driven out of equilibrium without breaking a spatial symmetry. Besides the application to biological systems, active matter serves as a paradigm to study the effect of broken time-reversal symmetry and nonequilibrium steady states in general [4,5]. Much progress has been made in the understanding of the properties of active matter by using active Brownian particles (ABPs) as a model system. ABPs violate time-reversal symmetry by consuming fuel to generate motion, often referred to as self-propulsion.

In this study we show that in the presence of a spacedependent magnetic field, a macroscopic flux emerges from a flux-free system of ABPs. This stands in marked contrast with

\footnotetext{
*sharma@ipfdd.de

Published by the American Physical Society under the terms of the Creative Commons Attribution 4.0 International license. Further distribution of this work must maintain attribution to the author(s) and the published article's title, journal citation, and DOI.
}

similar phenomena in condensed matter such as the classical Hall effect [6], which requires an explicitly broken symmetry: a macroscopic velocity vector in addition to the symmetry breaking due to the magnetic field vector. Figure 1 shows an example of the effect of the Lorentz force on ABPs for a Gaussian-shaped magnetic field that is radially symmetric in the $x y$ plane and points in the $z$ direction. The two signatures are clearly visible: high density where the magnetic field is high and fluxes in the direction perpendicular to the gradient of the magnetic field.

As a model we consider a single self-propelled, charged Brownian particle in three dimensions subjected to a spacedependent magnetic field $\boldsymbol{B}(\boldsymbol{r})$. The active motion of the particle is modeled as a force in the direction of the orientation of the particle specified by a unit vector $\boldsymbol{p}$ undergoing rotational diffusion. The dynamics are described by the following (Stratonovich) stochastic differential equations:

$$
m \frac{d \boldsymbol{v}(t)}{d t}=-\gamma \Gamma[\boldsymbol{r}(t)] \cdot \boldsymbol{v}(t)+f \boldsymbol{p}(t)+\sqrt{2 \gamma T} \boldsymbol{\xi}(t),
$$

and

$$
\frac{d \boldsymbol{r}(t)}{d t}=\boldsymbol{v}(t), \text { and } \frac{d \boldsymbol{p}(t)}{d t}=\sqrt{2 D_{r}} \boldsymbol{\eta}(t) \times \boldsymbol{p}(t),
$$

where $m$ is the particle mass, $\gamma$ is the friction coefficient, $f$ is the self-propulsion force, $T$ is the temperature in units such that the Boltzmann constant is unity and $D_{r}$ is the rotational diffusion constant. The matrix $\Gamma(\boldsymbol{r})=\mathbf{1}+\kappa(\boldsymbol{r}) M$, with $\kappa=q B(\boldsymbol{r}) / \gamma$ where $q$ is the charge of the particle and $M$ is a matrix such that $B(\boldsymbol{r}) M \cdot \boldsymbol{v}=\boldsymbol{B}(\boldsymbol{r}) \times \boldsymbol{v}$, and $B(\boldsymbol{r}) \equiv|\boldsymbol{B}(\boldsymbol{r})|$. The stochastic vectors $\boldsymbol{\xi}$ and $\boldsymbol{\eta}$ are Gaussian distributed with zero mean and autocorrelation $\left\langle\boldsymbol{\xi}(t) \boldsymbol{\xi}^{T}\left(t^{\prime}\right)\right\rangle=\left\langle\boldsymbol{\eta}(t) \boldsymbol{\eta}^{T}\left(t^{\prime}\right)\right\rangle=$ $\delta\left(t-t^{\prime}\right) \mathbf{1}$. Note that there is no direct coupling between the orientation and the magnetic field. The parameter $\kappa$, which we refer to as the diffusive Hall constant, is a dimensionless measure of the strength of the Lorentz force relative to the frictional force. When this parameter is comparable 


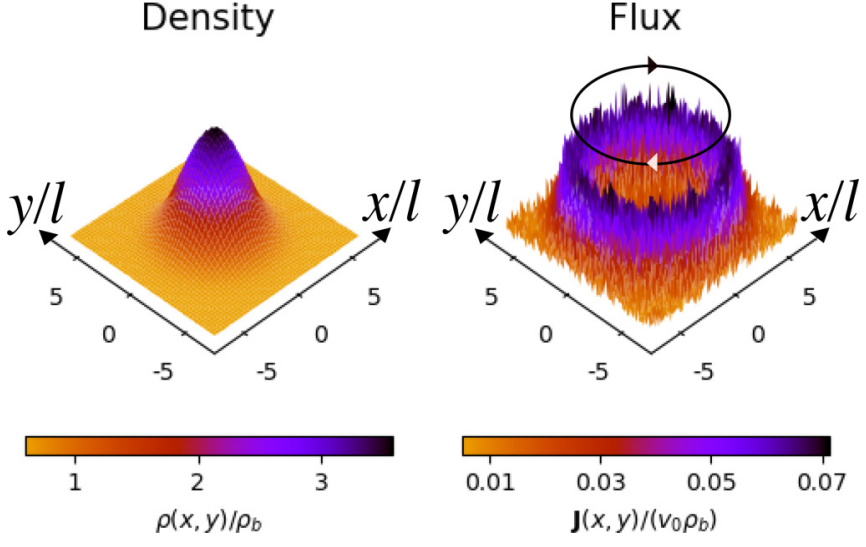

FIG. 1. Nonequilibrium steady state of noninteracting ABPs subjected to a Lorentz force. $l$ denotes the persistence length of the ABP, $q$ its charge, $\gamma$ its friction coefficient, $v_{0}$ its self-propulsion speed, and $\rho_{b}$ is the bulk density. The Gaussian shaped magnetic field is radially symmetric and points in the $z$ direction. The steady state is characterized by (i) an inhomogeneous density distribution and (ii) flux (indicated by the arrows) perpendicular to the gradient of the magnetic field. The flux is reminiscent of the Corbino effect in electrical conductors [7] and can be reversed by reversing the magnetic field. The data was obtained from Brownian dynamics simulations with a magnetic field $\boldsymbol{B}(\boldsymbol{r}) \propto \hat{\mathbf{e}}_{z} e^{-5\left(x^{2}+y^{2}\right)}$ and periodic boundary conditions.

to unity, the Lorentz force significantly affects the motion of the particle. As we show later, the Lorentz force may become comparable to the frictional force for a highly charged particle in strong magnetic fields. We start with Eq. (1) with the inertia term because in presence of a Lorentz force the overdamped equation of motion cannot be used to determine observables depending on the velocity process (such as flux) $[2,3,8]$. Equations (1) and (2) are also used for the Brownian dynamics (BD) simulations to verify the analytical results (see Appendix G).

To obtain the density, polarization and flux, we coarse grain the FPE corresponding to Eqs. (1) and (2) in two steps. First, we expand the probability density in powers of the mass and integrate out the velocity degrees of freedom [2,9]. This procedure is valid when the velocity autocorrelation time is the smallest time scale in the system, so when $m / \gamma \ll 1 / 2 D_{r}$, where $m / \gamma$ is the velocity autocorrelation time, $1 / 2 D_{r}$ is autocorrelation time of the orientation vector. Second, the orientational degrees of freedom are removed by a gradient expansion [10,11]. The gradient expansion yields accurate expressions if the gradients in the system are small compared to the persistence length of the ABP. These steps are explained in detail in Appendix C. This results in a FPE for the position variable alone:

$$
\frac{\partial}{\partial t} \rho(\boldsymbol{r}, t)=-\nabla \cdot \boldsymbol{j}(\boldsymbol{r}, t)
$$

where

$$
\boldsymbol{j}(\boldsymbol{r}, t)=\Gamma^{-1}(\boldsymbol{r}) \cdot\left[\frac{f}{\gamma} \overline{\boldsymbol{p}}(\boldsymbol{r}, t) \rho(\boldsymbol{r}, t)-\frac{T}{\gamma} \nabla \rho(\boldsymbol{r}, t)\right],
$$

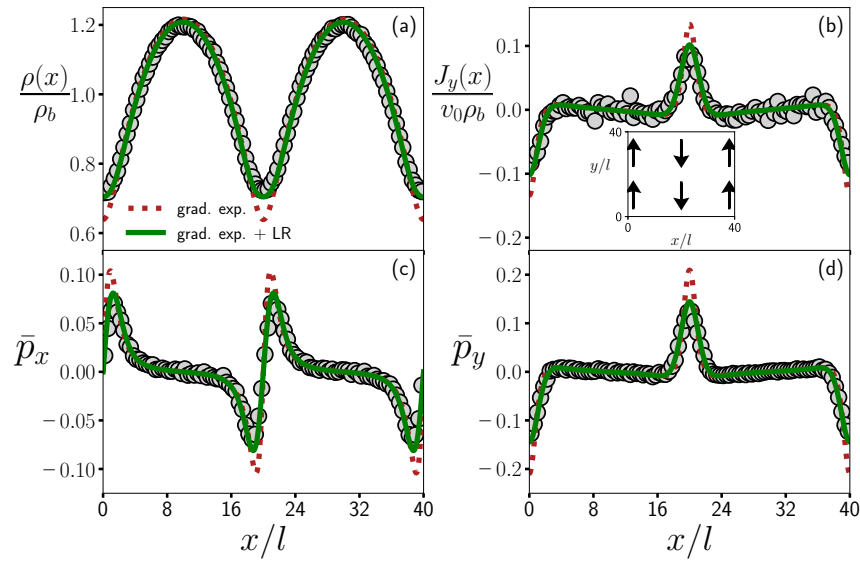

FIG. 2. (a) Density, (b) flux, and (c) and (d) orientation for $f=10, \kappa(x)=4 \sin (2 \pi x / 10), D_{r}=20, \gamma=1$, and $m=0.002$ in a system with periodic boundaries. The self-propulsion speed is $v_{0}=f / \gamma, l$ is the persistence length, and $\rho_{b}$ is the bulk density. The red dashed lines correspond to Eqs. (6)-(9). The green solid lines are the improved solutions, which use the linear-response calculations. The symbols represent the results from BD simulations. (a) The density is high where the magnitude of the magnetic field is large. This corresponds to accumulation in regions where the diffusion is hindered. (b) The flux in the system is proportional to the density and the polarization in the $y$ direction [see Eq. (9)]. The inset shows the flux lanes in the $x y$ plane. From (c) and (d) it is clear that the polarization is nonzero in a narrow space interval and changes rapidly; for example, from $x \approx 18 l$ to $x \approx 22 l$ the polarization rotates clockwise from the negative to the positive $x$ direction.

and

$$
\overline{\boldsymbol{p}}(\boldsymbol{r}, t)=-\frac{l}{3 \rho(\boldsymbol{r}, t)} \nabla \cdot\left[\Gamma^{-1}(\boldsymbol{r}) \rho(\boldsymbol{r}, t)\right],
$$

where $l=f / 2 D_{r} \gamma$ is the persistence length of the ABP, and $\overline{\boldsymbol{p}}(\boldsymbol{r}, t)$ is the polarization, which is defined as the average orientation per particle. Note that the matrix $\Gamma^{-1}$ in Eq. (4) is not symmetric, and therefore, $T \Gamma^{-1} / \gamma$ cannot be interpreted as a diffusion tensor [3].

Here we focus on the steady-state bulk behavior of the system and therefore use periodic boundary conditions in all directions. Furthermore, the analysis is restricted to a magnetic field pointing in the $z$ direction, and a magnitude that depends on $x$. Since the motion along the direction of the magnetic field is not affected by the Lorentz force, there is neither flux nor density variation along the $z$ direction in steady state. Because of the periodicity and equivalence of the positive and negative $x$ directions, in steady state there can be no flux in the $x$ direction either. The proof of the absence of flux in the $x$ direction is shown in Appendix $\mathrm{C}$ and has been corroborated by simulations.

By setting the $x$ component of the flux [Eq. (4)] to zero, we obtain the following expression for density:

$$
\rho(x)=N\left[1+\kappa^{2}(x)\right]^{\delta / 2},
$$

where $N^{-1}=\int_{0}^{L} d x\left[1+\kappa^{2}(x)\right]^{\delta / 2}$ and $\delta=\Delta T /(T+\Delta T)$, with $\Delta T=f^{2} / 6 D_{r} \gamma$, which is often called an effective temperature in effective theories of active matter [12]. In Fig. 2 we show the analytical results together with results from 
Brownian dynamics simulations for a system with $\kappa(x)=$ $4 \sin (2 \pi x / 10)$. We use a sinusoidal magnetic field because of its simple shape. Experimentally realizable magnetic fields are likely to have more complicated shapes; however, this does not change the conclusions of this study. The density is high where the magnitude of the magnetic field is large [see Fig. 2(a)]. Qualitatively, the accumulation of particles in the high magnetic field region can be understood as follows. A magnetic field hinders the diffusive motion of Brownian particles because there is a force perpendicular to the velocity, which results in circular motion. For passive Brownian particles this means that on time scales longer than the velocity autocorrelation time the effect of a uniform magnetic field is a decrease of the diffusion constant in the plane perpendicular to the magnetic field. For a spatially varying magnetic field, the diffusion coefficient of particles is small in the region where magnetic field is large. Whereas, for passive particles, the steady-state density distribution is independent of the diffusion coefficient, active particles accumulate in the region of low swim speed, which correspond to regions of high friction, or in this case high magnetic field [4,13]. Passive Brownian particles in a space-dependent magnetic field have a uniform steady-state density (a Boltzmann distribution with a uniform potential). A system of ABPs with a homogeneous activity also has a uniform steady-state density. However, the combination of a space-dependent magnetic field and uniform activity results in an inhomogeneous steady-state density. An inhomogeneous density without a space-dependent potential means that the density does not follow a Boltzmann distribution, a hallmark of nonequilibrium systems.

The polarization can be calculated by inserting Eq. (6) in Eq. (5), which yields

$$
\bar{p}_{x}(x)=\frac{(2-\delta) l}{3} \frac{\kappa(x) \kappa^{\prime}(x)}{\left[1+\kappa^{2}(x)\right]^{2}},
$$

for the $x$ component, and

$$
\bar{p}_{y}(x)=-\frac{l}{3} \frac{\kappa^{\prime}(x)}{\left[1+\kappa^{2}(x)\right]^{2}}\left[1-(1-\delta) \kappa^{2}(x)\right]
$$

for the $y$ component, where $\kappa^{\prime}(x)=\frac{d \kappa(x)}{d x}$. So there is polarization in the plane perpendicular to the magnetic field; see Eqs. (7) and (8) and Figs. 2(c) and 2(d).

In the $y$ direction there can be no density gradients because of the translation symmetry. This implies that the contribution to the flux in the $y$ direction coming from a density gradient along this direction is zero; however, the contribution from the polarization is not zero, resulting in a flux in the $y$ direction [see Fig. 2(b)]. This flux can be calculated by using the solution for the density [Eq. (6)] as input to the $y$ component of Eq. (4), yielding

$$
j_{y}(x)=\rho(x) \frac{f}{\gamma} \bar{p}_{y}(x) .
$$

A few remarks are in order: First, $j_{y}(x) / \rho(x)$ is the macroscopic velocity of the ABP and $f / \gamma$ is the intrinsic microscopic velocity. While the intrinsic velocity leads to random motion on large time scales, the macroscopic, field-induced motion persists on any time scale. Thus the polarization [Eq. (8)] gives the fraction of the swim force that is converted into macroscopic directed motion. Second, the orientation and flux in the $y$ direction are perpendicular to the gradient of the magnetic field. Third, the expressions for the density, flux, and polarization can be improved significantly by combining the gradient expansion with linear-response calculations of the polarization; see Appendix F.

There is no direct coupling between the magnetic field and the polarization [see Eq. (1) and Eq. (2)], and the inhomogeneous density along the $x$ direction is not a result of the polarization. Both the polarization and the inhomogeneous density result from a kinetic filtering mechanism. The swim force increases the active particle's velocity in the direction of its orientation. The Lorentz force rotates the velocity vector. The effect of this rotation is to make the velocity vector point in a direction different from its orientation. This leads to a decrease in the swim speed of the particle, that is, a decrease of the average velocity in the direction of the orientation, which is equivalent to reducing the effect of the active force. From this it follows that one of the effects of a spatially varying magnetic field on active particles is similar to subjecting them to an inhomogeneous activity field, for which it is well known that the steady-state density distribution scales inversely with the active force. A particle with an orientation antiparallel to the gradient of the magnetic field can move more easily towards regions of lower magnetic field. These effects result in a force imbalance between regions of small and large magnetic fields. In steady state the force imbalance is canceled by the gradient in the chemical potential due to the increase of density in the high magnetic region [13]. However, the analogy with an inhomogeneously active ABP only explains the inhomogeneous density, and it does not explain the steady-state fluxes in the magnetic system.

The steady-state bulk fluxes are a key feature of this nonequilibrium steady state of ABPs subjected to Lorentz force. These fluxes are unique in the sense that the underlying mechanism is independent of interparticle interactions [14], aligning torques [15], sliding motion along asymmetric walls [16-20], or a time- and space-dependent swim force [21,22]. Moreover, the direction of the flux is perpendicular to the gradient of the applied magnetic field. This particular property is what gives rise to the circulating steady-state flux shown in Fig. 1. The direction of the fluxes can be reversed by simply reversing the direction of the magnetic field.

In case of a finite system with walls, the bulk properties of the system are similar to that of an infinite system; however, there is also a flux parallel to the wall resulting in closed-loop fluxes (see Appendix A). A flux along walls has recently been investigated in the case of chiral active particle [23]. The flux along the wall induced by Lorentz force can possibly be explained by the same mechanism.

\section{ACTIVE DIFFUSION UNDER LORENTZ FORCE.}

Here we investigate the mean-squared displacement (MSD) of an ABP subjected to a space-dependent Lorentz force. Free ABPs have a swim speed of $f / \gamma$ [24]; therefore, on short time scales the motion of ABPs is ballistic and is the same in the three directions: $\left\langle\Delta x(t)^{2}\right\rangle=\left\langle\Delta y(t)^{2}\right\rangle=$ $\left\langle\Delta z(t)^{2}\right\rangle=(f t / \gamma)^{2}$, where $\Delta x(t)$ is $x(t)-x(0)$, and similarly for $\Delta y(t)$ and $\Delta z(t)$. The diffusion parallel to the 
magnetic field orientation is unaffected by the field. This implies that the diffusion coefficient in the $z$ direction is $D_{z}=(T+\Delta T) / \gamma$. Since a magnetic field hinders the motion in the plane perpendicular to its direction, the diffusion in the $x$ and $y$ directions is reduced. For an inhomogeneous magnetic field, the spatially varying diffusion coefficient in the $x$ and $y$ directions can be obtained from the symmetric part of $\Gamma^{-1}$ as $D_{x}(x)=D_{y}(x)=D_{z} /\left[1+\kappa(x)^{2}\right]$. On a length scale larger than the period of the magnetic field, the space dependence of the diffusion constant becomes irrelevant, and one can approximate the effective diffusion constant for MSD in the $x$ direction by averaging over space: $D_{x}^{(\text {eff })}=\int_{0}^{L} d x \rho(x) D_{x}(x)$, where $L$ is the period of the magnetic field.

There are three time scales in the system. The shortest time scale, $\tau_{1}$, is the transition from ballistic to diffusive motion in the $x$ direction, which can be obtained from $\left(f \tau_{1} / \gamma\right)^{2}=$ $2 D_{x}^{\text {(eff) }} \tau_{1}$, and is $\tau_{1}=2 \gamma^{2} D_{x}^{\text {(eff) }} / f^{2}$. The second time scale, $\tau_{2}$, is the transition from ballistic to diffusive motion in the $z$ direction, which can be calculated from $\left(f \tau_{2} / \gamma\right)^{2}=2 D_{z} \tau_{2}$ as $\tau_{2}=2 \gamma^{2} D_{z} / f^{2}$. The largest time scale, $\tau_{3}$, is the transition to diffusive motion in the $y$ direction. Because the net flux in the system is zero, the motion in the $y$ direction becomes diffusive on the same time scale as the time it takes to diffuse from on lane to another. This time scale can be calculated from $2 D_{x}^{\text {(eff) }} \tau_{3}=(L / 2)^{2}$ as $\tau_{3}=L^{2} / 8 D_{x}^{\text {(eff) }}$.

The MSD in the $x$ and $y$ directions become sub-ballistic on the same time scale $\left(\tau_{1}\right)$; however, in the $y$ direction the diffusive regime starts later at $\tau_{3}$, meaning that there is a super diffusive regime that persists over several decades in time. Even though the magnetic field hinders diffusion in both $x$ and $y$ direction, on long time scales the MSD in the $y$ direction is larger than that of the $x$ direction. Figure 3 shows that when $t>\tau_{3}$ the MSD in the $y$ direction has the same diffusion coefficient as the $z$ direction.

\section{PASSIVE DIFFUSION OF TRACER PARTICLE.}

Direct observation of active flux lanes might be difficult in experiments because the Lorentz force on an ABP is small. To overcome this limitation, we consider the cumulative effect of these lanes on a passive tracer particle (see inset of Fig. 3). The lane velocity is $v_{y}=j_{y} / \rho=v_{0} \bar{p}_{y} \approx v_{0}^{2} q|\nabla B| /\left(2 D_{r} \gamma\right)$, where $v_{0}=f / \gamma$ is the self-propulsion speed, $|\nabla B|$ is the (maximum) gradient of the magnetic field, and $q$ the total charge on the ABP. Although the lane velocity here is calculated for the special case of sinusoidal magnetic field, the same could be done for any other choice of magnetic field as long as there are large gradients at the location of interest. To estimate the charge of an ABP, we consider a Janus-shaped particle with half of its surface grafted with polymer chains, and the other half serving as the propulsion engine [25-29]. The total charge on such an $\mathrm{ABP}$ is $q=2 \pi R^{2} \sigma_{b} \lambda e$, where $R$ is the radius of the ABP, $\sigma_{b}$ is the grafting density, $\lambda$ is the number of charges per polymer chain, and $e$ is the elementary charge. Employing Stokes relations for the rotational diffusion coefficient $D_{r}$ and $\gamma$, together with the parameters $k_{b} T=$ $298 \mathrm{kgm}^{2} \mathrm{~s}^{-2}$, a high grafting density of $\sigma_{b}=1 \mathrm{~nm}^{-2}$ and highly charged chains with $\lambda=100,|\nabla B|=10 T / \mathrm{cm}, L \sim$ $1 \mathrm{~cm}, v_{0} \approx 3 \mu \mathrm{m} \mathrm{s}^{-1}$ and $R \approx 2 \mu \mathrm{m}$, we obtain $v_{y} \approx 2.5 \times$ $10^{-3} \mu \mathrm{m} \mathrm{s}^{-1}$. This would result in a drift of tracer particle

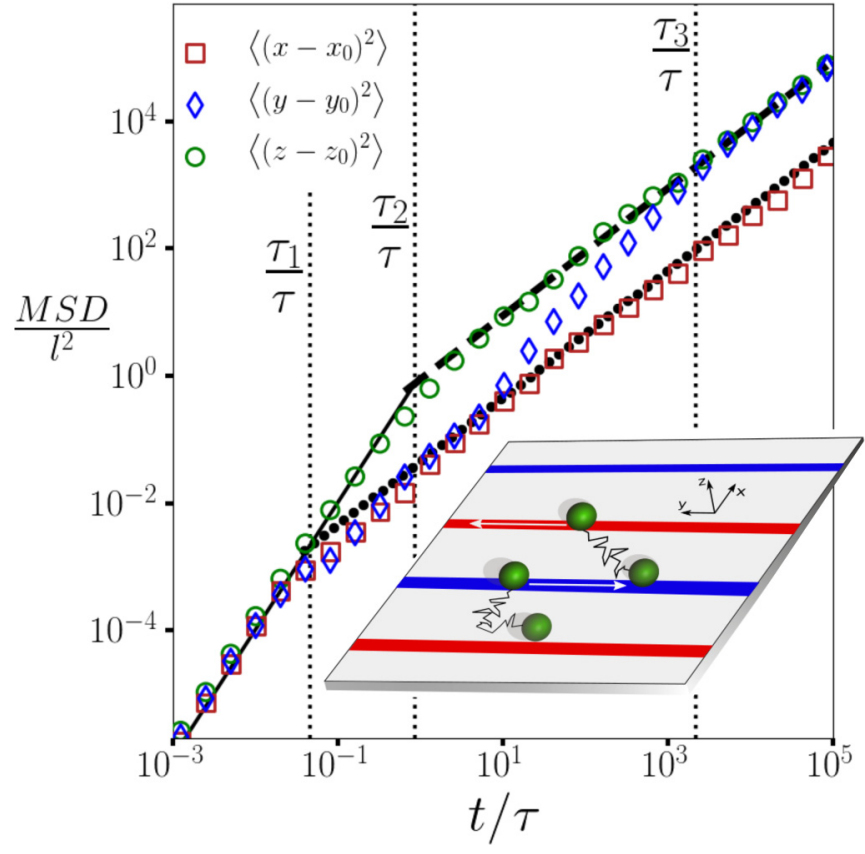

FIG. 3. The mean-square displacement of an ABP with $f=20$, $\kappa(x)=8 \sin (2 \pi x / 10), \gamma=1$, and $D_{r}=20 . l$ is the persistence length of the ABP, and $\tau=1 / 2 D_{r}$ its orientational correlation time. Symbols represent results from BD simulations. Diffusion parallel to the magnetic field orientation is unaffected by the field. For $t>\tau_{2}$, the motion in the $z$ direction is diffusive (dashed line) with diffusion constant $D_{z}$. The motion of an ABP along the $x$ is also diffusive for $t>\tau_{1}$ (dotted line), albeit with a smaller diffusion coefficient $D_{x}^{\text {(eff) }}$. For $t<\tau_{2}$, the motion along $y$ is same as along $x$. The time scale of the transition from ballistic to diffusive motion in the $z$ direction is $\tau_{2}$. However, at long times $t>\tau_{3}$, the rate of diffusion along $y$ becomes the same as that of a freely diffusing ABP. The time scale of the transition to diffusive motion in the $y$ direction is $\tau_{3}$. At intermediate times $\tau_{2}<t<\tau_{3}$, the motion along $y$ is super diffusive. Inset shows a schematic of a passive tracer particle diffusing in the $x y$ plane. The flux lanes are shown in blue and red. The particle diffuses from one lane to the other shown as zigzag lines. Inside a lane, the particle drifts along with the local velocity of the lane shown as white arrows. This results in anisotropic diffusion of the particle.

along the flux lane of the order of millimeter per day. Note that with these parameters the diffusive Hall constant $\kappa=$ 0.1 , implying that Lorentz force become comparable to the frictional force on the particle.

It is assumed above that the tracer particle does not diffuse out of the lane in a day. For a $\mu \mathrm{m}$ sized tracer particle, with diffusion coefficient $D_{p} \approx 10^{-13} \mathrm{~m}^{2} / \mathrm{s}$ and lane width $d=L /(2 \pi) \approx 0.1 \mathrm{~cm}$, this is indeed the case. On longer time scales, the diffusion of the tracer particle will become anisotropic and can be characterized by the ratio of its MSDs in the $y$ and $x$ directions, which is $r_{a}=1+\left(v_{y}^{2} d^{4}\right) /\left(D_{p}^{2} L^{2}\right) \approx$ 2 for the parameters above. Similar problems have been studied in different contexts, such as the diffusion of a passive particle in presence of random velocity fields [30] and the motion of polymer chains in random layered flows [31].

Note that the total charge of the polyelectrolyte brush is compensated by counter-ions arising from the dissociation of the charged groups of polymers in water. At a high salt 

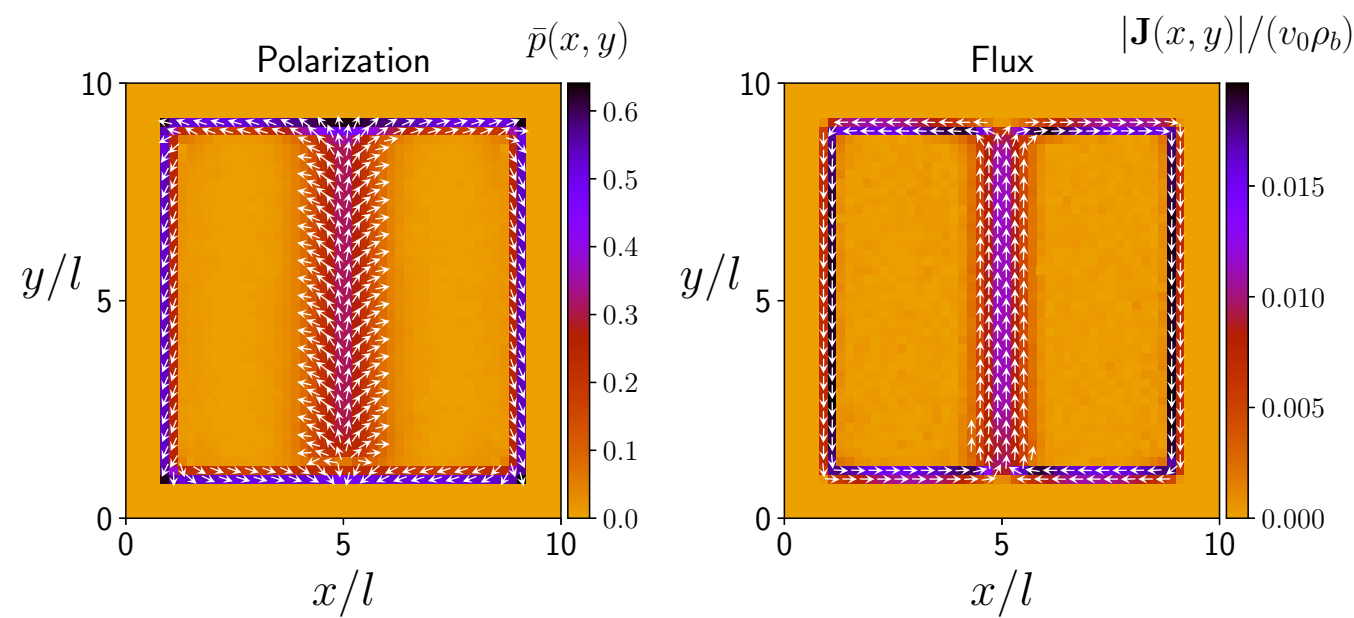

FIG. 4. Polarization and flux in a confined system. The results are obtained from Brownian dynamics simulations with $f=20, \kappa(x)=$ $8 \sin (2 \pi x / 5), \gamma=1, D_{r}=20$, and $m=0.002$ in a system with walls at $x=0, y=0, x=10$, and $y=10$. The self-propulsion speed of the ABPs is $v_{0}=f / \gamma, l$ is their persistence length and $\rho_{b}$ is the bulk density. The walls are modeled by a WCA potential.

level in the solution the counter-ions are quasifree $[32,33]$. Furthermore, their exchange dynamics is much faster than the Lorentz-induced drift. A superior aspect of external magnetic field is that it is not screened by electric charges. Moreover, with large magnetic field gradients becoming accessible [34], brush decorated ABPs may be a promising experimental system.

\section{CONCLUSION}

In this study we showed that the Lorentz force gives rise to nontrivial behavior in a system of noninteracting ABPs. More research is needed to investigate how the Lorentz force affects other phenomena in active matter, such as the motilityinduced phase transition [35], and its behavior in complex environments [36]. Due to the relation between the Coriolis force in noninertial reference frames and the Lorentz force [37], the results from this study could be of interest to the study of ABPs in noninertial frames [38]. Furthermore, we expect that similar macroscopic effects could be induced by a magnetic field in a system with topological constraints such as active particles confined to a non-Euclidean surface [39].

\section{APPENDIX A: FLUX LOOPS IN NONEQUILIBRIUM STEADY STATE WITH WALLS}

The main text focused on bulk behavior, and periodic boundary conditions were used everywhere. Here we briefly explore a closed system with the same shape of the magnetic field as in Fig. 2 in the main text and comment on the effect of the boundaries; see Fig. 4.

In the bulk the density, polarization and flux in the system are similar to those in a system without walls. The polarization of ABPs near a wall is know to be directed towards the wall [40]; in this system, however, the orientation near the walls along the $y$ direction is directed downwards. This is in line with the prediction if one considers the gradient of the magnetic field at those positions. With the walls there are additional polarization effects: there is also polarization parallel to the walls in the $x$ direction. The polarization along the walls is related to the flux along the walls. Polarization and flux along the walls have also been observed in the case of chiral particles [23]. One can induce multiple lanes of bulk flux with opposing direction by decreasing the period of the magnetic field.

\section{APPENDIX B: SMALL-MASS LIMIT}

In this section we show how to derive the small-mass limit of the Fokker-Planck equation corresponding to the equation of motion [Eqs. (1) and (2) in the main text]. This limit is based on an expansion in powers of the mass of the particles, and subsequantly integrating out the velocity degrees of freedom. The resulting Fokker-Planck equation only depends on the spatial and orienational degrees of freedom and hold for time scales larger than the velocity autocorrelation time.

The equations of motion are

$$
\begin{gathered}
m \frac{d \boldsymbol{v}(t)}{d t}=-\gamma \Gamma[\boldsymbol{r}(t)] \cdot \boldsymbol{v}(t)+f \boldsymbol{p}(t)+\sqrt{2 \gamma T} \boldsymbol{\xi}(t), \\
\frac{d \boldsymbol{r}(t)}{d t}=\boldsymbol{v}(t)
\end{gathered}
$$

and

$$
\frac{d \boldsymbol{p}(t)}{d t}=\sqrt{2 D_{r}} \boldsymbol{\eta}(t) \times \boldsymbol{p}(t),
$$

where $\Gamma(\boldsymbol{r})=1+\kappa(\boldsymbol{r}) M$, with $\kappa(\boldsymbol{r})=q B(r) / \gamma$ and

$$
M=\left[\begin{array}{ccc}
0 & -1 & 0 \\
1 & 0 & 0 \\
0 & 0 & 0
\end{array}\right] .
$$

In order to obtain the small-mass limit of the FPE corresponding to these stochastic differential equations one can use the method described in Chap. 10 of Ref. [9]. This was done for a single passive particle in a homogeneous magnetic field in Ref. [2]. We start with the FPE corresponding to Eqs. (B2), (1), and (B3) for the time evolution of the probability density $P(t) \equiv P(\boldsymbol{r}, \boldsymbol{v}, \boldsymbol{p}, t)[41]$

$$
\frac{\partial}{\partial t} P(t)=\left(L_{\mathrm{rev}}+L_{\mathrm{irr}}+L_{r}+L_{a}\right) P(t),
$$


where the time-evolution operator has been split up in a reversible part

$$
L_{\mathrm{rev}} P(t)=-\boldsymbol{v} \cdot \nabla_{\boldsymbol{r}} P(t)+\frac{1}{m} B(\boldsymbol{r}) \nabla_{\boldsymbol{v}} \cdot[M \cdot \boldsymbol{v} P(t)],
$$

an irreversible part without the part coming from Eq. (B3)

$$
L_{\mathrm{irr}} P(t)=\frac{\gamma}{m} \nabla_{v} \cdot\left[\boldsymbol{v} P(t)+\frac{T}{m} \nabla_{v} P(t)\right],
$$

the part representing the rotation of the orientation vector

$$
L_{r} P(t)=D_{r} \mathcal{R}^{2} P(t),
$$

with the rotation operator $\mathcal{R}=\boldsymbol{p} \times \nabla_{\boldsymbol{p}}$ [42], and the active, nonequilibrium operator coming from the self-propulsion

$$
L_{a} P(t)=-\frac{f}{m} \boldsymbol{p} \cdot \nabla_{v} P(t) .
$$

Equation (B5) is equivalent to

$$
\frac{\partial}{\partial t} \bar{P}(t)=\left(\bar{L}_{\mathrm{rev}}+\bar{L}_{\mathrm{irr}}+\bar{L}_{r}+\bar{L}_{a}\right) \bar{P}(t),
$$

where

$$
\bar{P}(t)=P(t) R(v)^{-1 / 2}
$$

and

$$
\bar{L}=R(\boldsymbol{v})^{-1 / 2} L R(v)^{1 / 2},
$$

where $L$ can be any of the operators in Eq. (B5), and

$$
R(\boldsymbol{v})=\left(\frac{m}{2 \pi T}\right)^{3 / 2} e^{-\frac{m}{2 T} \boldsymbol{v}^{2}}
$$

is the solution to $L_{\mathrm{irr}} R(v)=0$, normalized such that the integral over $v$ is one. The transformed operators are

$$
\begin{gathered}
\bar{L}_{\mathrm{rev}}=-\sqrt{\frac{T}{m}} \nabla_{\boldsymbol{r}} \cdot\left(\boldsymbol{b}^{\dagger}+\boldsymbol{b}\right)+\frac{q}{m} \boldsymbol{B}(\boldsymbol{r}) \cdot\left(\boldsymbol{b}^{\dagger} \times \boldsymbol{b}\right), \\
\bar{L}_{\mathrm{irr}}=-\frac{\gamma}{m} \boldsymbol{b}^{\dagger} \cdot \boldsymbol{b}, \\
\bar{L}_{r}=L_{r}
\end{gathered}
$$

and

$$
\bar{L}_{a}=\frac{f}{\sqrt{T m}} \boldsymbol{p} \cdot \boldsymbol{b}^{\dagger},
$$

where $\boldsymbol{b}=\sqrt{T / m} \nabla_{\boldsymbol{v}}+\frac{1}{2} \sqrt{m / T} \boldsymbol{v}$ and $\boldsymbol{b}^{\dagger}=-\sqrt{T / m} \nabla_{\boldsymbol{v}}+$ $\frac{1}{2} \sqrt{m / T} \boldsymbol{v}$.

The eigenfunctions of the operator $b_{\alpha}^{\dagger} b_{\alpha}$, where $\alpha$ is $x, y$, or $z$, are

$$
\psi_{0}\left(v_{\alpha}\right)=\left(\frac{m}{2 \pi T}\right)^{1 / 4} e^{-\frac{m}{4 T} v_{\alpha}^{2}}
$$

and

$$
\psi_{n}\left(v_{\alpha}\right)=\frac{\psi_{0}\left(v_{\alpha}\right)}{\sqrt{n ! 2^{n}}} H_{n}\left(\sqrt{\frac{m}{2 T}} v_{\alpha}\right) \text { for } n>0,
$$

where $H_{n}$ are Hermite polynomials. The operators $b_{\alpha}^{\dagger}$ and $b_{\alpha}$ are the raising and lowering operators of the eigenfunctions: $b_{\alpha}^{\dagger} \psi_{n}\left(v_{\alpha}\right)=\sqrt{n+1} \psi_{n+1}\left(v_{\alpha}\right)$ and $b_{\alpha} \psi_{n}\left(v_{\alpha}\right)=\sqrt{n} \psi_{n-1}\left(v_{\alpha}\right)$. Because the eigenfunctions are orthonormal,

$$
\int_{-\infty}^{\infty} d x \psi_{n}(x) \psi_{m}(x)=\delta_{n, m},
$$

they can be used to expand $\bar{P}(t)$ :

$$
\bar{P}(t)=\sum_{n_{x}, n_{y}, n_{z}=0}^{\infty} c_{n_{x}, n_{y}, n_{z}} \psi_{n_{x}}\left(v_{x}\right) \psi_{n_{y}}\left(v_{y}\right) \psi_{n_{z}}\left(v_{z}\right),
$$

where $c_{n_{x}, n_{y}, n_{z}} \equiv c_{n_{x}, n_{y}, n_{z}}(\boldsymbol{r}, \boldsymbol{p}, t)$.

The probability density for the position and orientation, $Q(t) \equiv Q(\boldsymbol{r}, \boldsymbol{p}, t)$, is given by the first expansion coefficient:

$$
\begin{gathered}
Q(t)=\int d \boldsymbol{v} P(t) \\
=\int d \boldsymbol{v} \bar{P}(t) \psi_{0}\left(v_{x}\right) \psi_{0}\left(v_{y}\right) \psi_{0}\left(v_{z}\right) \\
=c_{0,0,0} .
\end{gathered}
$$

The translational flux,

$$
\boldsymbol{J}(\boldsymbol{r}, \boldsymbol{p}, t)=\int d \boldsymbol{v} \boldsymbol{v} P(\boldsymbol{r}, \boldsymbol{v}, \boldsymbol{p}, t),
$$

can be evaluated by using Eqs. (B11), (B21), and $v_{\alpha} \psi_{0}\left(v_{\alpha}\right)=$ $\sqrt{\frac{T}{m}} \psi_{1}\left(v_{\alpha}\right)$

$$
\boldsymbol{J}(\boldsymbol{r}, \boldsymbol{p}, t)=\sqrt{T / m} \boldsymbol{c}_{1}(\boldsymbol{r}, t) .
$$

Using this, together with the definition of the flux in orientation space

$$
\boldsymbol{J}_{\text {rot }}(\boldsymbol{r}, \boldsymbol{p}, t)=-D_{r} \mathcal{R} Q(t),
$$

one obtains the following equation for the time evolution of the probability density:

$$
\partial_{t} Q(t)=-\nabla_{\boldsymbol{r}} \cdot \boldsymbol{J}-\mathcal{R} \cdot \boldsymbol{J}_{\text {rot }} .
$$

Next, we need an expression for the $c_{1}$ in terms of $Q(t)$. We are interested in the case where the magnetic field is oriented perpendicular to the gradient of the field, and take this to be the $z$ direction, so $\boldsymbol{B}(\boldsymbol{r})=B(\boldsymbol{r}) \hat{z}$. Equation (B10) together with the orthonormality of the eigenfunctions yields an hierarchy of equations for the functions $c_{n_{x}, n_{y}, n_{z}}$ called a Brinkman hierarchy [43]:

$$
\begin{aligned}
\frac{\partial}{\partial t} c_{n_{x}, n_{y}, n_{z}} & \\
= & -\frac{\gamma}{m} c_{n_{x}, n_{y}, n_{z}}\left(n_{x}+n_{y}+n_{z}\right) \\
& -\boldsymbol{D} \cdot\left[\begin{array}{l}
\sqrt{n_{x}+1} c_{n_{x}+1, n_{y}, n_{z}} \\
\sqrt{n_{y}+1} c_{n_{x}, n_{y}+1, n_{z}} \\
\sqrt{n_{z}+1} c_{n_{x}, n_{y}, n_{z}+1}
\end{array}\right]-\hat{\boldsymbol{D}} \cdot\left[\begin{array}{c}
\sqrt{n_{x}} c_{n_{x}-1, n_{y}, n_{z}} \\
\sqrt{n_{y}} c_{n_{x}, n_{y}-1, n_{z}} \\
\sqrt{n_{z}} c_{n_{x}, n_{y}, n_{z}-1}
\end{array}\right] \\
& +\frac{q B(\boldsymbol{r})}{m} \sqrt{n_{x}\left(n_{y}+1\right)} c_{n_{x}-1, n_{y}+1, n_{z}} \\
& -\frac{q B(\boldsymbol{r})}{m} \sqrt{\left(n_{x}+1\right) n_{y}} c_{n_{x}+1, n_{y}-1, n_{z}}+D_{r} \mathcal{R}^{2} c_{n_{x}, n_{y}, n_{z}},
\end{aligned}
$$

where $\boldsymbol{D}=\sqrt{\frac{T}{m}} \nabla_{\boldsymbol{r}}$ and $\hat{\boldsymbol{D}}=\sqrt{\frac{T}{m}} \nabla_{\boldsymbol{r}}-\frac{1}{\sqrt{T m}} f \boldsymbol{p}$.

The equation governing the time evolution of the first expansion coefficient is

$$
\frac{\partial}{\partial t} c_{0,0,0}=-\boldsymbol{D} \cdot \boldsymbol{c}_{1}+D_{r} \boldsymbol{\mathcal { R }}^{2} c_{0,0,0}
$$


where $c_{1}=\left(c_{1,0,0}, c_{0,1,0}, c_{0,0,1}\right)^{T}$. This is of course the same as Eq. (B28). The equation for $\boldsymbol{c}_{1}(t)$ is again a differential equation in $t$, which can be solved after a Laplace transformation:

$$
\begin{aligned}
& \widetilde{\Gamma}_{m}^{(1)}(s) \cdot \widetilde{\boldsymbol{c}}_{1}(s) \\
& \quad=\frac{m}{\gamma}\left[\boldsymbol{c}_{1}(0)+D_{r} \boldsymbol{\mathcal { R }}^{2} \widetilde{\boldsymbol{c}}_{1}(s)-D_{1,2} \cdot \widetilde{\boldsymbol{c}}_{2}(s)-\hat{\boldsymbol{D}} \cdot \widetilde{\boldsymbol{c}}_{0,0,0}(s)\right],
\end{aligned}
$$

where the tilde indicates a Laplace transformation from the variable $t$ to $s, D_{1,2}$ is a matrix of which the entries are linearly proportional to $D_{x}, D_{y}$, or $D_{z}, c_{2}(t)$ is a vector of which the elements are all $c_{n_{x}, n_{y}, n_{z}}$ such that $n_{x}+n_{y}+n_{z}=2$, and

$$
\widetilde{\Gamma}_{m}^{(1)}(s)=\left[\begin{array}{ccc}
1+s \frac{m}{\gamma} & -\kappa(\boldsymbol{r}) & 0 \\
\kappa(\boldsymbol{r}) & 1+s \frac{m}{\gamma} & 0 \\
0 & 0 & 1+s \frac{m}{\gamma}
\end{array}\right] .
$$

Note that $\widetilde{\Gamma}_{m}^{(1)}(s)=\Gamma(\boldsymbol{r})+O(m)$. The Laplace transformation of the equation for $c_{2}(t)$ is

$$
\begin{aligned}
& \Gamma_{m}^{(2)}(\boldsymbol{r}, s) \cdot \tilde{\boldsymbol{c}}_{2}(s) \\
& \quad=\frac{m}{\gamma}\left[\boldsymbol{c}_{2}(0)+D_{r} \boldsymbol{\mathcal { R }}^{2} \widetilde{\boldsymbol{c}}_{2}-D_{2,3} \cdot \tilde{\boldsymbol{c}}_{3}-\hat{D}_{2,1} \cdot \tilde{\boldsymbol{c}}_{1}\right],
\end{aligned}
$$

where $\widetilde{\Gamma}_{m}^{(2)}$ is a matrix of which the entries are at least of order $m^{0}, D_{2,3}$ is a matrix whose elements are proportional to $D_{x}$, $D_{y}$ or $D_{z}, \hat{D}_{2,1}$ is a matrix whose elements are proportional to $\hat{D}_{x}, \hat{D}_{y}$, or $\hat{D}_{z}$, and $c_{3}(t)$ is a vector of which the elements are all $c_{n_{x}, n_{y}, n_{z}}$ such that $n_{x}+n_{y}+n_{z}=3$.

By only retaining the zeroth-order contribution in $m$ to Eq. (B31), one obtains an equation that is independent of $v$ and holds for $s m / \gamma \ll 1$ (that is, for times longer than the velocity autocorrelation time). The vector $\boldsymbol{D}$ is of order $m^{-1 / 2}$, so only the terms of order $m^{1 / 2}$ of $c_{1}$ are needed, which means that one does not need the matrices $D_{1,2}, D_{2,3}$, and $\hat{D}_{2,1}$. Equation (B34) can be used to replace the $c_{2}$ in Eq. (B32), which, after inverting the Laplace transformation and only retaining terms of order $m^{1 / 2}$ or lower, becomes

$$
\Gamma \cdot c_{1}(t)=-\frac{m}{\gamma} \hat{D} c_{0,0,0}+O(m)
$$

which gives for the translational flux

$$
\boldsymbol{J}(\boldsymbol{r}, \boldsymbol{p}, t)=-\frac{1}{\gamma} \Gamma^{-1} \cdot\left(T \nabla_{\boldsymbol{r}}-f \boldsymbol{p}\right) Q(t)+O\left(m^{1 / 2}\right),
$$

where

$$
\Gamma^{-1}(\boldsymbol{r})=\mathbf{1}-\frac{\kappa(\boldsymbol{r})}{1+\kappa^{2}(\boldsymbol{r})} M+\frac{\kappa^{2}(\boldsymbol{r})}{1+\kappa^{2}(\boldsymbol{r})} M^{2} .
$$

The result for the translational flux together with Eqs. (B24) and (B31) gives following Fokker-Planck equation:

$$
\partial_{t} Q(t)=\frac{1}{\gamma} \nabla \cdot\left[\Gamma^{-1} \cdot\left(T \nabla_{\boldsymbol{r}}-f \boldsymbol{p}\right) Q(t)\right]+D_{r} \mathcal{R}^{2} Q(t) .
$$

\section{APPENDIX C: GRADIENT EXPANSION}

The goal of the gradient expansion is to integrate out the orientational degrees of freedom of the probability density
$Q(\boldsymbol{r}, \boldsymbol{p}, t)$ and obtain a drift-diffusion equation for the probability density $\rho(\boldsymbol{r}, t)$ of the position variable $[11,44]$. This expansion is based on a decomposition of the probability density $Q(\boldsymbol{r}, \boldsymbol{p}, t)$ in spherical harmonics:

$$
Q(\boldsymbol{r}, \boldsymbol{p}, t)=\rho+\boldsymbol{\sigma} \cdot \boldsymbol{p}+\boldsymbol{\tau}:(\boldsymbol{p p}-\mathbf{1} / 3)+\Omega,
$$

where the scalar $\rho$, the vector $\sigma$ and the matrix $\tau$ are function of $\boldsymbol{r}$ and $t$. These functions are linear combinations of the projection of $Q$ on, respectively, the zeroth, first, and second spherical harmonic, and $\Omega$ is the projection onto the third- and higher-order spherical harmonics. Using this decomposition in Eqs. (B27), (B28), and (B36) and integrating over the orientational degrees of freedom gives

$$
\frac{\partial}{\partial t} \rho=-\nabla_{\boldsymbol{r}} \cdot \boldsymbol{j}
$$

where

$$
\boldsymbol{j}(\boldsymbol{r}, t)=-\frac{1}{\gamma} \Gamma^{-1} \cdot\left[T \nabla_{r} \rho-\frac{f}{3} \boldsymbol{\sigma}\right]
$$

is the flux in position space. An equation for $\sigma$ can be obtained by multiplying Eq. (B28) by $\boldsymbol{p}$ and then integrating over the orientational degrees of freedom. This gives

$$
\begin{aligned}
\frac{\partial}{\partial t} \sigma_{a}= & \frac{T}{\gamma} \partial_{b}\left(\Gamma_{b c}^{-1} \partial_{c} \sigma_{a}\right) \\
& -\frac{f}{\gamma} \partial_{b}\left(\frac{2}{5} \Gamma_{b c}^{-1} \tau_{c a}+\Gamma_{b a}^{-1} \rho\right)-2 D_{r} \sigma_{a},
\end{aligned}
$$

where $a, b$, and $c$ are the indices of the vectors and matrices, and $\Gamma_{a b}^{-1}$ denotes the $a b$ component of the matrix $\Gamma^{-1}$. Similarly, an equation for $\tau$ can be obtained by multiplying Eq. (B28) by $\boldsymbol{p} \boldsymbol{p}-\mathbf{1} / 3$ and then integrating over the orientational degrees of freedom:

$$
\begin{aligned}
\frac{\partial}{\partial t} \tau_{a b}= & \frac{T}{\gamma} \partial_{c}\left(\Gamma_{c d}^{-1} \partial_{d} \tau_{a b}\right) \\
& -\frac{f}{2 \gamma} \partial_{c}\left(\Gamma_{c a}^{-1} \sigma_{b}+\Gamma_{c b}^{-1} \sigma_{a}-\frac{2}{3} \Gamma_{c d}^{-1} \delta_{a b}\right) \\
& -6 D_{r} \tau_{a b}+\partial_{c} \Upsilon_{a b c},
\end{aligned}
$$

where the $\partial_{c} \Upsilon_{c a b}$ comes from the projection on to the thirdand higher-order harmonics. Equations (C2), (C4), (C5) are exact, but not closed since $\Upsilon$ is not specified.

We now consider the limit in which the gradients in the system are small in comparison to the persistence length of the $\mathrm{ABP}[10,11]$. The relaxation time of $\rho$ is of order $\sim(\nabla)^{-1}$, whereas all the other harmonics relax in times of order $\sim 1$. This implies that in the limit of small gradients, $\rho$ is the slowest mode. We thus assume that the time derivative of $\sigma$ and terms with both a $\sigma$ and a $\nabla$ can be neglected. This yields

$$
\sigma=-\frac{f}{2 D_{r} \gamma} \nabla_{\boldsymbol{r}} \cdot\left[\Gamma^{-1} \rho\right]
$$

which is related to the polarization by $\bar{p}=\frac{1}{3} \sigma / \rho$. With this expansion the flux becomes

$$
\boldsymbol{j}(\boldsymbol{r}, t)=-\frac{f^{2}}{6 D_{r} \gamma^{2}} \Gamma^{-1} \cdot \nabla \cdot\left(\Gamma^{-1} \rho\right)-\frac{T}{\gamma} \Gamma^{-1} \cdot \nabla \rho .
$$


In a system with periodic boundary conditions and a magnetic field that depends on the $x$ coordinate only, the flux in the $x$ direction must be a constant in steady state. The flux in the $x$ direction is

$$
\begin{aligned}
j_{x}= & -\frac{T+\Delta T}{\gamma} \frac{1}{1+\kappa(x)^{2}} \partial_{x} \rho(x) \\
& -\frac{\Delta T}{\gamma} \frac{1}{2}\left(\partial_{x} \frac{1}{1+\kappa(x)^{2}}\right) \rho,
\end{aligned}
$$

where $\left[\Gamma^{-1}\right]_{x \beta}\left[\Gamma^{-1}\right]_{x \beta}=\left[\Gamma^{-1}\right]_{x x}=1 /\left(1+\kappa(x)^{2}\right)$ was used, and $\Delta T=f^{2} /\left(6 D_{r} \gamma\right)$. This equation can be solved for $\rho(x)$ :

$$
\begin{aligned}
\rho(x)= & {\left[1+\kappa(x)^{2}\right]^{\delta / 2}\left(A\left(x_{0}\right)\right.} \\
& \left.-j_{x} \frac{\gamma}{T+\Delta T} \int_{x_{0}}^{x} d x^{\prime}\left[1+\kappa\left(x^{\prime}\right)^{2}\right]^{1-\delta / 2}\right),
\end{aligned}
$$

where $x_{0}$ is some reference position, $A\left(x_{0}\right)$ is a constant that depends on $x_{0}$ and $\delta=\Delta T /(T+\Delta T)$. For system of size $L$ in the $x$ direction, and $\kappa(x)=\kappa(x+L)$, so the magnetic field repeats itself $L / n$ where $n$ is an integer,

$$
\begin{aligned}
\rho(x)= & \rho(x+L) \\
= & {\left[1+\kappa(x)^{2}\right]^{\delta / 2}\left\{A\left(x_{0}\right)\right.} \\
& \left.-j_{x} \frac{\gamma}{T+\Delta T} \int_{x_{0}}^{x+L} d x^{\prime}\left[1+\kappa\left(x^{\prime}\right)^{2}\right]^{1-\delta / 2}\right\} \\
= & \rho(x)-j_{x} \frac{\gamma}{T+\Delta T} \int_{x}^{L} d x^{\prime}\left[1+\kappa\left(x^{\prime}\right)^{2}\right]^{1-\delta / 2} .
\end{aligned}
$$

Therefore

$$
j_{x} \frac{\gamma}{T+\Delta T} \int_{x}^{L} d x^{\prime}\left[1+\kappa(x)^{2}\right]^{1-\delta / 2}=0,
$$

which is only satisfied for $j_{x}=0$. So in steady state there is no flux in the $x$ direction and the steady-state density is

$$
\rho(x) \propto\left[1+\kappa(x)^{2}\right]^{\delta / 2},
$$

where the proportionality constant is determined by the normalization of the density. Using this density to calculate the $y$ component of the steady-state flux gives

$$
j_{y}(x)=\frac{f}{\gamma} \bar{p}_{y}(x) \rho(x) .
$$

The steady-state polarization in the $x$ and $y$ directions can be determined from Eq. (C6) and the density:

$$
\bar{p}_{x}=-\frac{(\delta-2) l}{3} \frac{\kappa(x) \kappa^{\prime}(x)}{\left[1+\kappa(x)^{2}\right]^{2}},
$$

and

$$
\bar{p}_{y}=-\frac{l}{3} \frac{\kappa^{\prime}(x)}{\left[1+\kappa(x)^{2}\right]^{2}}\left[1+(1-\delta) \kappa(x)^{2}\right] .
$$

\section{APPENDIX D: LINEAR-RESPONSE THEORY}

Here we develop the linear-response theory that is used in the next section to calculate the orientation. The orientation calculated in this way can be used to improve the solution from the gradient expansion from the previous section; see Appendix F.

Linear-response theory for ABPs has been developed in Ref. [45] to study the average swim speed, and was later applied to a system of interacting ABPs with inhomogeneous activity to study the density profile [13] and flux [22]. Here we adapt the linear-response theory for ABPs and apply it to an charged ABP in a space-dependent magnetic field.

$$
\frac{\partial}{\partial t} Q(t)=\left(\mathcal{L}_{d}+\mathcal{L}_{r}+\mathcal{L}_{a}\right) Q(t)
$$

where

$$
\mathcal{L}_{d} Q(t)=\frac{T}{\gamma} \nabla_{\boldsymbol{r}} \cdot\left[\Gamma^{-1} \cdot \nabla_{\boldsymbol{r}} Q(t)\right]
$$

is the operator corresponding to the particle's diffusive behavior, $\mathcal{L}_{r}=L_{r}$ is the rotational time-evolution operator and

$$
\mathcal{L}_{a} Q(t)=-\frac{f}{\gamma} \nabla_{\boldsymbol{r}} \cdot\left[\Gamma^{-1} \cdot \boldsymbol{p} Q(t)\right]
$$

is the nonequilibrium time-evolution operator. Next, we split the probability density $Q(t)$ in an equilibrium $\left(Q_{\text {eq }}\right)$ and a nonequilibrium $[\delta Q(t)]$ part, where the equilibrium part is the steady-state solution to Eq. (D1) with $f=0$, which is constant in space. The equation for the nonequilibrium part is

$$
\frac{\partial}{\partial t} \delta Q(t)=\left(\mathcal{L}_{d}+\mathcal{L}_{r}+\mathcal{L}_{a}\right) \delta Q(t)+\mathcal{L}_{a} Q_{\mathrm{eq}},
$$

because $\partial_{t} Q_{\mathrm{eq}}=\left(\mathcal{L}_{d}+\mathcal{L}_{r}\right) Q_{\mathrm{eq}}=0$.

We are interested in nonequilibrium steady-state averages, and therefore assume that the system started out at $t=-\infty$ in the equilibrium state. The previous equation can be integrated from $t=-\infty$ to $t=0$ by treating $\mathcal{L}_{a} Q_{\text {eq }}$ as an inhomogeneity:

$$
\delta Q_{s s}=-\frac{f}{\gamma} \int_{0}^{\infty} d \tau e^{\tau\left(\mathcal{L}_{d}+\mathcal{L}_{r}+\mathcal{L}_{a}\right)} Q_{\mathrm{eq}}\left[\nabla_{\boldsymbol{r}} \cdot \Gamma^{-1}(\boldsymbol{r})\right] \cdot \boldsymbol{p},
$$

where $\delta Q_{s s}=\delta Q(t=\infty)$ is the steady-state solution of the nonequilibrium part of the probability density. This equation is exact and can be used to calculate the steady-state nonequilibrium average of a generic function $g=g(\boldsymbol{r}, \boldsymbol{p})$ :

$$
\begin{gathered}
\langle g\rangle^{\boldsymbol{r}, \boldsymbol{p}}=\int d \boldsymbol{r} \int d \boldsymbol{p} Q_{s s}(\boldsymbol{r}, \boldsymbol{p}) g \\
=\langle g\rangle_{\mathrm{eq}}^{\boldsymbol{r}, \boldsymbol{p}}-\frac{f}{\gamma} \int_{0}^{\infty} d t\left\langle\left[\nabla_{\boldsymbol{r}} \cdot \Gamma^{-1}(\boldsymbol{r})\right] \cdot \boldsymbol{p} e^{t\left(\mathcal{L}_{d}^{\dagger}+\mathcal{L}_{r}^{\dagger}+\mathcal{L}_{a}^{\dagger}\right)} g\right\rangle_{\mathrm{eq}}^{\boldsymbol{r}, \boldsymbol{p}},
\end{gathered}
$$

where $Q_{s s}(\boldsymbol{r}, \boldsymbol{p})=Q_{\mathrm{eq}}+\delta Q_{s s}(\boldsymbol{r}, \boldsymbol{p})$ is the nonequilibrium steady-state probability density, the super script $\boldsymbol{r}, \boldsymbol{p}$ denotes the variables that are averaged over, the subscript $e q$ indicates that the average is taken with respect to the equilibrium distribution, and a $\dagger$ indicates the adjoint of an operator. The adjoint operators acting on a function $g$ are

$$
\begin{gathered}
\mathcal{L}_{d}^{\dagger} g(\boldsymbol{r}, \boldsymbol{p})=\frac{T}{\gamma} \nabla_{\boldsymbol{r}} \cdot\left(\Gamma^{-1, T} \cdot \nabla_{r} g\right), \\
\mathcal{L}_{r}^{\dagger} g(\boldsymbol{r}, \boldsymbol{p})=D_{r} \nabla_{\boldsymbol{p}} \times\left\{\boldsymbol{p} \cdot\left[\boldsymbol{\nabla}_{\boldsymbol{p}} \times(\boldsymbol{p} g)\right]\right\},
\end{gathered}
$$


and

$$
\mathcal{L}_{a}^{\dagger} g(\boldsymbol{r}, \boldsymbol{p})=\frac{f}{\gamma} \boldsymbol{p} \cdot\left(\Gamma^{-1, T} \cdot \nabla_{\boldsymbol{r}} g\right),
$$

where the superscript $T$ indicates a transpose.

The response of the system to the activity up to linear order in $f$ is obtained by removing from the full time-evolution operator the nonequilibrium operator $\mathcal{L}_{a}^{\dagger}$ :

$$
\langle g\rangle_{l r}^{\boldsymbol{r}, \boldsymbol{p}}=\langle f\rangle_{\mathrm{eq}}^{\boldsymbol{r}, \boldsymbol{p}}-\frac{f}{\gamma} \int_{0}^{\infty} d t\left\langle\left[\nabla_{\boldsymbol{r}} \cdot \Gamma^{-1}(\boldsymbol{r})\right] \cdot \boldsymbol{p} e^{t\left(\mathcal{L}_{d}^{\dagger}+\mathcal{L}_{r}^{\dagger}\right)} g\right\rangle_{\mathrm{eq}}^{\boldsymbol{r}, \boldsymbol{p}} .
$$

This Green-Kubo relation defines the active transport coefficient $\alpha$ corresponding to an functions $g$ by $\alpha=$ $\lim _{f \rightarrow 0}\left(\langle g\rangle_{l r}-\langle g\rangle_{\text {eq }}\right) / f$. Equation (D11) shows that only quantities that are odd functions of $\boldsymbol{p}$ have a nonzero linear response because the angular average in equilibrium any function of an odd power of $\boldsymbol{p}$ yields zero by symmetry.

\section{APPENDIX E: POLARIZATION FROM LINEAR-RESPONSE THEORY}

Here we calculate the polarization using the general linearresponse theory developed in the previous Appendix. The steady-state polarization is defined as the average orientation per particle:

$$
\overline{\boldsymbol{p}}\left(\boldsymbol{r}^{\prime}\right)=\frac{\left\langle\boldsymbol{p} \delta\left(\boldsymbol{r}^{\prime}-\boldsymbol{r}\right)\right\rangle^{\boldsymbol{r}, \boldsymbol{p}}}{\rho\left(\boldsymbol{r}^{\prime}\right)},
$$

where $\rho\left(\boldsymbol{r}^{\prime}\right)$ is the average steady-state density, which is the same as the steady-state probability density of particles as a function of the position alone. Because the density operator $\hat{\rho}\left(\boldsymbol{r}^{\prime}\right)=\delta\left(\boldsymbol{r}^{\prime}-\boldsymbol{r}\right)$ is independent of the orientation, the density has no linear response to the activity; therefore, when one only considers contributions up to linear order in the swim force to the polarization, the position-dependent density can be replaced by the bulk density $\rho_{b}$.

The orientation up to linear order in $f$ can be calculated using Eq. (D11) to evaluate the average in Eq. (E1):

$\overline{\boldsymbol{p}}^{(1)}\left(\boldsymbol{r}^{\prime}\right)=-\frac{f}{\rho_{b} \gamma} \int_{0}^{\infty} d t\left\langle\left(\nabla_{\boldsymbol{r}} \cdot \Gamma^{-1}\right) \cdot \boldsymbol{p} e^{t\left(\mathcal{L}_{d}^{\dagger}+\mathcal{L}_{r}^{\dagger}\right)} \boldsymbol{p} \delta\left(\boldsymbol{r}^{\prime}-\boldsymbol{r}\right)\right\rangle_{\mathrm{eq}}^{\boldsymbol{r}, \boldsymbol{p}}$,

where the superscript (1) indicates that it is correct up to first order in the self-propulsion force $f$. Because in equilibrium the position is not correlated with the orientation, one can integrate out the orientational degrees of freedom in the average. The orientational integral in the equilibrium average in Eq. (E2) is

$$
\left\langle\boldsymbol{p} e^{t \mathcal{L}_{r}^{\dagger}} \boldsymbol{p}\right\rangle_{\text {eq }}^{p}=\langle\boldsymbol{p}(0) \boldsymbol{p}(t)\rangle,
$$

where the $\boldsymbol{p}(t)$ is the solution of the stochastic process resulting from Eq. (B3) with initial orientation $p(0)$, and the angle brackets indicate an average over realizations of the noise. This is the autocorrelation function for Brownian motion on a unit sphere and can be calculated by expanding the corresponding FPE in spherical harmonics [12] as

$$
\left\langle\boldsymbol{p}(t) \boldsymbol{p}\left(t^{\prime}\right)\right\rangle=\frac{1}{3} e^{-2 D_{r}\left|t-t^{\prime}\right|} \mathbf{1} .
$$

With the result for the autocorrelation of $p(t)$, Eq. (E2) becomes

$$
\overline{\boldsymbol{p}}^{(1)}\left(\boldsymbol{r}^{\prime}\right)=-f \int_{0}^{\infty} d t \frac{e^{-2 D_{r} t}}{3 \rho_{b} \gamma}\left\langle\left(\nabla_{\boldsymbol{r}} \cdot \Gamma^{-1}\right) e^{t \mathcal{L}_{d}^{\dagger}} \delta\left(\boldsymbol{r}^{\prime}-\boldsymbol{r}\right)\right\rangle_{\mathrm{eq}}^{\boldsymbol{r}},
$$

where average is taken with respect to the equilibrium probability density for the position variable. By adding an other integral, one can take the gradient of $\Gamma^{-1}$ out of the average:

$$
\begin{aligned}
\overline{\boldsymbol{p}}^{(1)}\left(\boldsymbol{r}^{\prime}\right)= & -f \int_{0}^{\infty} d t \frac{e^{-2 D_{r} t}}{3 \rho_{b} \gamma} \int d \boldsymbol{r}^{\prime \prime}\left[\nabla_{\boldsymbol{r}^{\prime \prime}} \cdot \Gamma^{-1}\left(\boldsymbol{r}^{\prime \prime}\right)\right] \\
& \times\left\langle\delta\left(\boldsymbol{r}^{\prime \prime}-\boldsymbol{r}\right) e^{t \mathcal{L}_{d}^{\dagger}} \delta\left(\boldsymbol{r}^{\prime}-\boldsymbol{r}\right)\right\rangle_{\mathrm{eq}}^{\boldsymbol{r}},
\end{aligned}
$$

which can be written as

$$
\overline{\boldsymbol{p}}^{(1)}\left(\boldsymbol{r}^{\prime}\right)=f \int_{0}^{\infty} d t \int d \boldsymbol{r}^{\prime \prime} \chi\left(\boldsymbol{r}^{\prime}, \boldsymbol{r}^{\prime \prime}, t\right),
$$

where the response function $\chi\left(\boldsymbol{r}^{\prime}, \boldsymbol{r}^{\prime \prime}, t\right)$ is

$$
\chi\left(\boldsymbol{r}^{\prime}, \boldsymbol{r}^{\prime \prime}, t\right)=-\frac{1}{3 \gamma} e^{-2 D_{r} t} G\left(\boldsymbol{r}^{\prime}, \boldsymbol{r}^{\prime \prime}, t\right) \nabla_{\boldsymbol{r}^{\prime \prime}} \cdot \Gamma^{-1}\left(\boldsymbol{r}^{\prime \prime}\right),
$$

and

$$
G\left(\boldsymbol{r}^{\prime}, \boldsymbol{r}^{\prime \prime}, t\right)=\frac{1}{\rho_{b}}\left\langle\delta\left(\boldsymbol{r}^{\prime \prime}-\boldsymbol{r}\right) e^{t \mathcal{L}_{d}^{\dagger}} \delta\left(\boldsymbol{r}^{\prime}-\boldsymbol{r}\right)\right\rangle_{\mathrm{eq}}^{\boldsymbol{r}}
$$

is similar to a Van Hove function, but with a space-dependent diffusion constant $[46,47]$. In the case of a space-independent diffusion tensor, Eq. (E9) would become a Van Hove function after integrating out one of the coordinates.

By working out what $\nabla_{\boldsymbol{r}} \cdot \Gamma^{-1}(\boldsymbol{r})$ is, one finds that there is no orientation along the direction of the magnetic field. Working out the derivative gives

$$
\begin{aligned}
\nabla_{\boldsymbol{r}} \cdot \Gamma^{-1}(\boldsymbol{r})=- & \frac{1-\kappa(\boldsymbol{r})^{2}}{\left[1+\kappa(\boldsymbol{r})^{2}\right]^{2}} \nabla_{\boldsymbol{r}} \kappa(\boldsymbol{r}) \cdot M \\
& +\frac{2 \kappa(\boldsymbol{r}) \kappa^{\prime}(\boldsymbol{r})}{\left[1+\kappa(\boldsymbol{r})^{2}\right]^{2}} \nabla_{\boldsymbol{r}} \kappa(\boldsymbol{r}) \cdot M^{2}
\end{aligned}
$$

Without loss of generality one can consider the case where the magnetic field is oriented along the $z$ direction. In this case the matrices $M$ and $M^{2}$ are

$$
M=\left[\begin{array}{ccc}
0 & -1 & 0 \\
1 & 0 & 0 \\
0 & 0 & 0
\end{array}\right] \text { and } M^{2}=\left[\begin{array}{ccc}
-1 & 0 & 0 \\
0 & -1 & 0 \\
0 & 0 & 0
\end{array}\right] \text {, }
$$

so clearly there is no orientation along the direction of the magnetic field.

The operator $\mathcal{L}_{d}^{\dagger}$ acting on $\boldsymbol{r}$ in Eq. (E6) has the same effect as acting with the adjoint on $\boldsymbol{r}^{\prime}$ (see, for example, Chap. 4.2 of Ref. [9]); therefore

$$
\begin{aligned}
\overline{\boldsymbol{p}}^{(1)}\left(\boldsymbol{r}^{\prime}\right)= & -f \int_{0}^{\infty} d t \frac{e^{-2 D_{r} t}}{3 \rho_{b} \gamma} \int d \boldsymbol{r}^{\prime \prime}\left[\nabla_{\boldsymbol{r}^{\prime \prime}} \cdot \Gamma^{-1}\left(\boldsymbol{r}^{\prime \prime}\right)\right] \\
& \times\left\langle\delta\left(\boldsymbol{r}^{\prime \prime}-\boldsymbol{r}\right) e^{t \mathcal{L}_{d}\left(\boldsymbol{r}^{\prime}\right)} \delta\left(\boldsymbol{r}^{\prime}-\boldsymbol{r}\right)\right\rangle_{\mathrm{eq}}^{\boldsymbol{r}} .
\end{aligned}
$$

where

$$
\mathcal{L}_{d}\left(\boldsymbol{r}^{\prime}\right)=T \nabla_{\boldsymbol{r}^{\prime}} \cdot \Gamma^{-1}\left(\boldsymbol{r}^{\prime}\right) \cdot \nabla_{\boldsymbol{r}^{\prime}},
$$


and

$$
\frac{1}{\rho_{b}}\left\langle\delta\left(\boldsymbol{r}^{\prime \prime}-\boldsymbol{r}\right) \delta\left(\boldsymbol{r}^{\prime}-\boldsymbol{r}\right)\right\rangle^{\boldsymbol{r}}=\delta\left(\boldsymbol{r}^{\prime \prime}-\boldsymbol{r}^{\prime}\right) \frac{\rho\left(\boldsymbol{r}^{\prime}\right)}{\rho_{b}},
$$

was used. In the linear-response calculation of the orientation $\rho\left(\boldsymbol{r}^{\prime}\right)$ can be replaced with $\rho_{b}$.

Equation (E12) can be simplified to

$$
\begin{aligned}
\overline{\boldsymbol{p}}^{(1)}\left(\boldsymbol{r}^{\prime}\right)= & -f \int_{0}^{\infty} d t \frac{1}{3 \gamma} e^{-2 D_{r} t} e^{t \mathcal{L}_{d}\left(\boldsymbol{r}^{\prime}\right)} \\
& \times \int d \boldsymbol{r}^{\prime \prime} \delta\left(\boldsymbol{r}^{\prime \prime}-\boldsymbol{r}^{\prime}\right) \nabla_{\boldsymbol{r}^{\prime \prime}} \cdot \Gamma^{-1}\left(\boldsymbol{r}^{\prime \prime}\right),
\end{aligned}
$$

and after the $\boldsymbol{r}^{\prime \prime}$ integral this becomes

$$
\overline{\boldsymbol{p}}^{(1)}(\boldsymbol{r})=-f \int_{0}^{\infty} d t \frac{1}{3 \gamma} e^{-2 D_{r} t} e^{t \mathcal{L}_{d}(\boldsymbol{r})} \nabla_{\boldsymbol{r}} \cdot \Gamma^{-1}(\boldsymbol{r}) .
$$

Multiplying both sides of this equation by $2 D_{r}-\mathcal{L}_{d}(\boldsymbol{r})$ results in

$$
\begin{aligned}
& {\left[2 D_{r}-\mathcal{L}_{d}(\boldsymbol{r})\right] \overline{\boldsymbol{p}}^{(1)}(\boldsymbol{r})} \\
& \quad=\frac{f}{3 \gamma} \int_{0}^{\infty} d t \frac{\partial}{\partial t} e^{-t\left[2 D_{r}-\mathcal{L}_{d}(\boldsymbol{r})\right]} \nabla_{\boldsymbol{r}} \cdot \Gamma^{-1}(\boldsymbol{r}),
\end{aligned}
$$

which is equivalent to the following differential equation:

$$
\left[2 D_{r}-\mathcal{L}_{d}(\boldsymbol{r})\right] \overline{\boldsymbol{p}}^{(1)}(\boldsymbol{r})=-\frac{f}{3 \gamma} \nabla_{\boldsymbol{r}} \cdot \Gamma^{-1}(\boldsymbol{r}) .
$$

This equation is the main result of this Appendix. Solutions can be obtained numerically, or by further approximations.

For large $D_{r}$ and a magnetic field with small gradients, the contribution from the operator $\mathcal{L}_{d}$ to the left-hand side of Eq. (E18) is negligible compared to the contribution coming from the part with $D_{r}$; therefore, one can approximate, in this case, the solution to this equation by

$$
\overline{\boldsymbol{p}}^{(1)}(\boldsymbol{r}) \approx-\frac{1}{3} l \nabla_{\boldsymbol{r}} \cdot \Gamma^{-1}(\boldsymbol{r}) .
$$

Note that this is the same Eqs. $(\mathrm{C} 14)$ and $(\mathrm{C} 15)$ without the terms of cubic order in $f$.

Even though the density does not have a linear response to the swim speed, the linear response calculations for the orientation can be used to calculate the density and fluxes [13]. First, one integrates out the orientational degrees of freedom from Eq. (B28):

$$
\frac{\partial}{\partial t} \rho(\boldsymbol{r}, t)=-\nabla_{\boldsymbol{r}} \cdot \boldsymbol{j}(\boldsymbol{r}, t)
$$

with

$$
\boldsymbol{j}(\boldsymbol{r}, t)=-\frac{1}{\gamma} \Gamma^{-1} \cdot\left[T \nabla_{\boldsymbol{r}}-f \overline{\boldsymbol{p}}(\boldsymbol{r}, t)\right] \rho(\boldsymbol{r}, t),
$$

where $\overline{\boldsymbol{p}}(\boldsymbol{r}, t) \equiv \frac{1}{\rho(\boldsymbol{r}, t)} \int d \boldsymbol{p} Q(\boldsymbol{r}, \boldsymbol{p}, t)$ is the polarization.

When the magnetic field is oriented in the $z$ direction and depends only on the $x$ coordinate, equating the steady-state flux in the $x$ direction to zero gives the following density:

$$
\rho^{(2)}(x) \propto \exp \left[\frac{f}{T} \int^{x} d x^{\prime} \bar{p}_{x}^{(1)}\left(x^{\prime}\right)+\kappa\left(x^{\prime}\right) \bar{p}_{y}^{(1)}\left(x^{\prime}\right)\right],
$$

where the proportionality constant is determined by normalization, and the superscript (2) indicates that it is correct up to second order in the swim force (there is no linear-order response in the density). With this density the steady-state flux in the $y$ direction can be calculated from Eq. (E21):

$$
j_{y}^{(2)}(x)=\frac{\rho_{b} f}{\gamma} \bar{p}_{y}^{(1)}(x)
$$

where $\rho_{b}$ is the bulk density.

\section{APPENDIX F: IMPROVED SOLUTION}

Only using the linear-order contributions in $f$ to the orientation gives poor agreement with simulations for the density; see Fig. 5. The terms of cubic order in $f$ contribute to the diffusion in the system, and by ignoring them there has to be a larger density gradient to compensate for the flux resulting from the orientation. This problem can be overcome by combining the first-order result from linear-response theory with the cubic terms from the gradient expansion.

Using the gradient expansion (Appendix C) we found that the flux is

$$
\boldsymbol{j}(\boldsymbol{r}, t)=-\Gamma^{-1} \cdot[f \rho \overline{\boldsymbol{p}}]-\frac{T}{\gamma} \Gamma^{-1} \cdot \nabla \rho,
$$

where $\overline{\boldsymbol{p}}=\overline{\boldsymbol{p}}(\boldsymbol{r}, t)$ is the average polarization at position $\boldsymbol{r}$, which is

$$
\begin{aligned}
\overline{\boldsymbol{p}}(\boldsymbol{r}, t) & =-\frac{l}{3 \rho} \nabla \cdot\left(\Gamma^{-1} \rho\right) \\
& =-\frac{l}{3} \nabla \cdot \Gamma^{-1}-\frac{l}{3} \Gamma^{-1, T} \cdot \nabla \ln \rho .
\end{aligned}
$$

The second term on the right-hand side is higher order in the swim force because a gradient in the density is at least second order. The first term on the right-hand side of this equation is proportional to the swim force and is equal to the linear-response solution in the limit of small gradients. However, Eq. (E18) gives the linear-order response of the polarization without assuming that the gradients are small. We therefore replace the linear-order term with the numerical solution to the linear-response equation for the polarization [Eq. (E18)] and rewrite the previous equation as

$$
\overline{\boldsymbol{p}}(\boldsymbol{r}, t)=\overline{\boldsymbol{p}}^{(1)}-\frac{l}{3} \Gamma^{-1, T} \cdot \nabla \ln \rho .
$$

When the magnetic field is oriented in the $z$ direction and varies in the $x$ direction, one can use this expression for the polarization together with the expression for the flux [Eq. (F1)] to obtain the steady-state density by equating the flux in the $x$ direction to zero:

$$
\rho(x)=N \exp \left[\frac{f}{T+\Delta T} \int_{0}^{x} d x^{\prime} \bar{p}_{x}^{(1)}\left(x^{\prime}\right)+\kappa(x) \bar{p}_{y}^{(1)}\left(x^{\prime}\right)\right],
$$

where $N$ is a normalization constant such that $\int_{0}^{L} d x \rho(x)=1$ and $L$ the size of the periodic box. Note that this expression is the same as the density calculated only using linear-response 

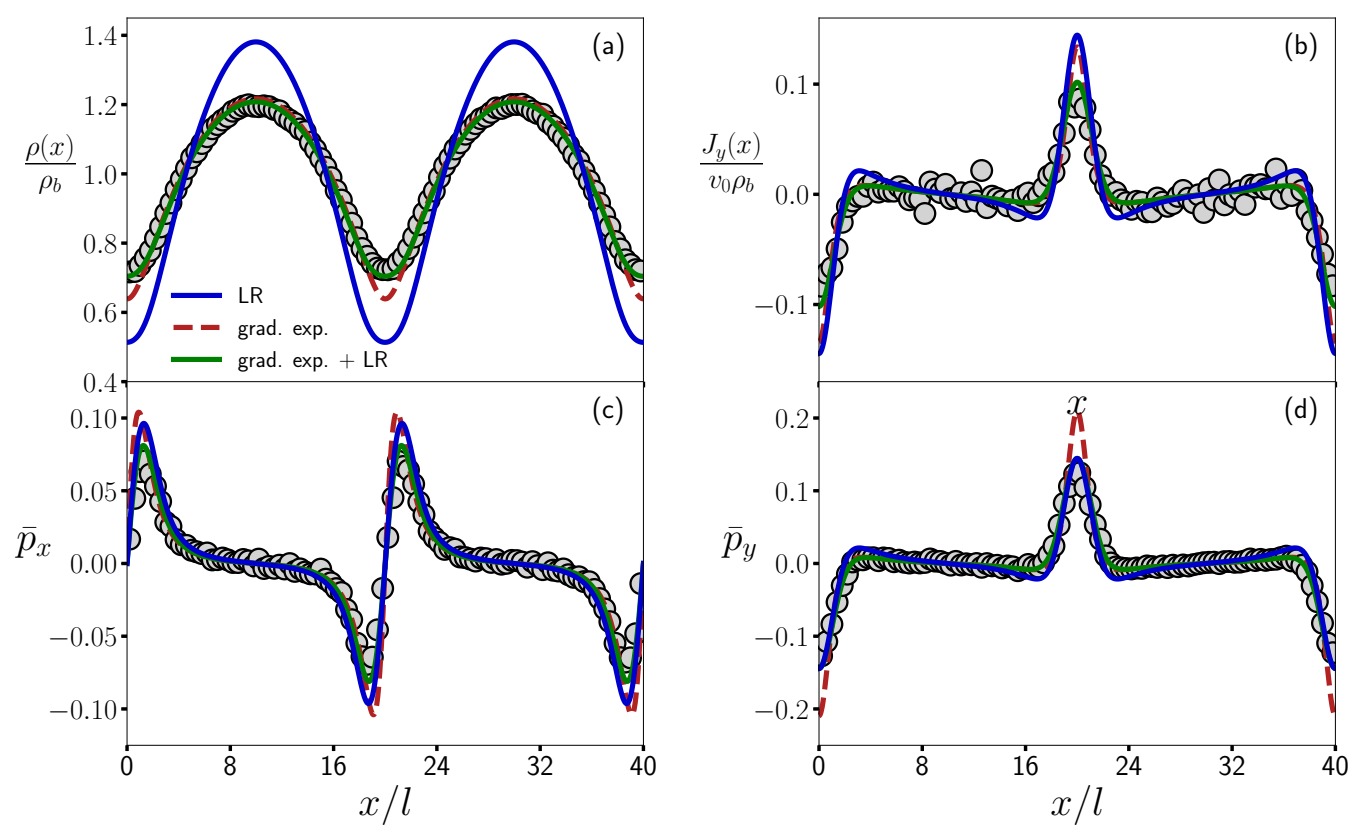

FIG. 5. (a) Density, (b) flux, and (c) and (d) orientation for $f=10, \kappa(x)=4 \sin (2 \pi x / 10), D_{r}=20, \gamma=1$ and $m=0.002$. The selfpropulsion speed is $v_{0}=f / \gamma, l$ is the persistence length, and $\rho_{b}$ is the bulk density. The blue solid lines correspond to the linear-response results [Eqs. (E19), (E22), and (E23)]. The red, dashed lines correspond to Eqs. (C12) to (C13). The green, solid lines are the improved solutions, which uses the linear-response calculations [Eqs. (F4)-(F7)]. The symbols represent the results from BD simulations. This is the same figure as Fig. 2 in the main text, but with the linear-response calculations included.

theory [Eq. (E22)], but with $T$ replaced by $T+\Delta T$. The flux in the $y$ direction can be calculated from Eq. (F1) together with the result from the density as

$$
j_{y}(x)=\frac{f}{\gamma} \rho \bar{p}_{y}^{(1)}+\frac{f \delta}{\gamma} \frac{\kappa}{1+\kappa^{2}}\left(\bar{p}_{x}^{(1)}-\kappa \bar{p}_{y}^{(1)}\right) .
$$

And finally, the density can be used in Eq. (F3) to obtain the polarization in the $x$ and $y$ directions in terms of the linearresponse polarization as

$$
\bar{p}_{x}(x)=\bar{p}_{x}^{(1)}-\frac{\delta}{1+\kappa^{2}}\left(\bar{p}_{x}^{(1)}+\kappa \bar{p}_{y}^{(1)}\right),
$$

and

$$
\bar{p}_{y}(x)=\bar{p}_{y}^{(1)}-\frac{\kappa \delta}{1+\kappa^{2}}\left(\bar{p}_{x}^{(1)}+\kappa \bar{p}_{y}^{(1)}\right) .
$$

\section{APPENDIX G: SIMULATION DETAILS AND NUMERICS}

The Brownian dynamics simulations were done by using the Euler algorithm to discretize the equations of motion in time [Eqs. (B2)-(B3)]. After each time increment of the orientation vector [Eq. (B3)], the vector is rescaled such that the length is always unity. For all simulations a time step of $\Delta t=$ $0.5 \times 10^{-6}$ was used. The flux in the system was obtained by sampling the velocity of the ABP. The linear-response equation for the orientation [Eq. (E18)] was integrated using the boundary value problem solver from the Scipy PYTHON library [48].
[1] P. Grasland-Mongrain, R. Souchon, F. Cartellier, A. Zorgani, J.-Y. Chapelon, C. Lafon, and S. Catheline, Imaging of Shear Waves Induced by Lorentz Force in Soft Tissues, Phys. Rev. Lett. 113, 038101 (2014).

[2] H. M. Chun, X. Durang, and J. D. Noh, Emergence of nonwhite noise in Langevin dynamics with magnetic Lorentz force, Phys. Rev. E 97, 032117 (2018).

[3] H. D. Vuijk, J. M. Brader, and A. Sharma, Anomalous fluxes in overdamped Brownian dynamics with Lorentz force, J. Stat. Mech.: Theory Exp. (2019) 063203.

[4] M. E. Cates, Diffusive transport without detailed balance in motile bacteria: Does microbiology need statistical physics? Rep. Prog. Phys. 75, 042601 (2012).
[5] E. Fodor, C. Nardini, M. E. Cates, J. Tailleur, P. Visco, and F. van Wijland, How Far from Equilibrium is Active Matter? Phys. Rev. Lett. 117, 038103 (2016).

[6] E. H. Hall, On a new action of the magnet on electric currents, Am. J. Mat. 2, 287 (1879).

[7] E. P. Adams, The hall and corbino effects, in Proc. Am. Phil. Soc 54, 47 (1915).

[8] S. Hottovy, A. McDaniel, G. Volpe, and J. Wehr, The smoluchowski-kramers limit of stochastic differential equations with arbitrary state-dependent friction, Commun. Math. Phys. 336, 1259 (2015).

[9] H. Risken, The Fokker-planck Equation, 2nd ed. (Springer, Berlin, 1989). 
[10] M. J. Schnitzer, Theory of continuum random walks and application to chemotaxis, Phys. Rev. E 48, 2553 (1993).

[11] M. E. Cates and J. Tailleur, When are active Brownian particles and run-and-tumble particles equivalent? Consequences for motility-induced phase separation, Europhys. Lett. 101, 20010 (2013).

[12] T. F.F. Farage, P. Krinninger, and J. M. Brader, Effective interactions in active Brownian suspensions, Phys. Rev. E 91, 042310 (2015).

[13] A. Sharma and J. M. Brader, Brownian systems with spatially inhomogeneous activity, Phys. Rev. E 96, 032604 (2017).

[14] J. Stenhammar, R. Wittkowski, D. Marenduzzo, and M. E. Cates, Light-induced self-assembly of active rectification devices, Sci. Adv. 2, e1501850 (2016).

[15] C. Lozano, B. Ten Hagen, H. Löwen, and C. Bechinger, Phototaxis of synthetic microswimmers in optical landscapes, Nature Commun. 7, 12828 (2016).

[16] N. Nikola, A. P. Solon, Y. Kafri, M. Kardar, J. Tailleur, and R. Voituriez, Active Particles with Soft and Curved Walls: Equation of State, Ratchets, and Instabilities, Phys. Rev. Lett. 117, 098001 (2016).

[17] J. Katuri, D. Caballero, R. Voituriez, J. Samitier, and S. Sanchez, Directed flow of micromotors through alignment interactions with micropatterned ratchets, ACS Nano 12, 7282 (2018).

[18] J. Rodenburg, S. Paliwal, M. De Jager, P. G. Bolhuis, M. Dijkstra, and R. Van Roij, Ratchet-induced variations in bulk states of an active ideal gas, J. Chem. Phys. 149, 174910 (2018).

[19] C. J. Olson Reichhardt and C. Reichhardt, Ratchet effects in active matter systems, Annu. Rev. Condens. Matter Phys. 8, 51 (2017).

[20] R. Di Leonardo, L. Angelani, G. Ruocco, V. Iebba, M. P. Conte, S. Schippa, F. De Angelis, F. Mecarini, and E. Di Fabrizio, A bacterial ratchet motor, Proc. Natl. Acad. Sci. 107, 9541 (2010).

[21] A. Geiseler, P. Hänggi, F. Marchesoni, C. Mulhern, and S. Savel'Ev, Chemotaxis of artificial microswimmers in active density waves, Phys. Rev. E 94, 012613 (2016).

[22] H. Merlitz, H. D. Vuijk, J. Brader, A. Sharma, and J.-U. Sommer, Linear response approach to active Brownian particles in time-varying activity fields, J. Chem. Phys. 148, 194116 (2018).

[23] L. Caprini and U. M. B. Marconi, Active chiral particles under confinement: surface currents and bulk accumulation phenomena, Soft Matter 15, 2627 (2019).

[24] H. Merlitz, C. Wu, and J.-U. Sommer, Directional transport of colloids inside a bath of self-propelling walkers, Soft Matter 13, 3726 (2017).

[25] S. Jiang, Q. Chen, M. Tripathy, E. Luijten, K. S. Schweizer, and S. Granick, Janus particle synthesis and assembly, Adv. Mater. 22, 1060 (2010).

[26] A. Walther and A. H. E. Müller, Janus particles: Synthesis, selfassembly, physical properties, and applications, Chem. Rev. 113, 5194 (2013).

[27] I. Buttinoni, G. Volpe, F. Kümmel, G. Volpe, and C. Bechinger, Active brownian motion tunable by light, J. Phys.: Condens. Matter 24, 284129 (2012).
[28] S. Samin and R. van Roij, Self-Propulsion Mechanism of Active Janus Particles in Near-Critical Binary Mixtures, Phys. Rev. Lett. 115, 188305 (2015).

[29] A. Würger, Self-Diffusiophoresis of Janus Particles in Near-Critical Mixtures, Phys. Rev. Lett. 115, 188304 (2015).

[30] J. P. Bouchaud, A. Georges, J. Koplik, A. Provata, and S. Redner, Superdiffusion in Random Velocity Fields, Phys. Rev. Lett. 64, 2503 (1990).

[31] J.-U. Sommer and A. Blumen, Polymer chains in random layered flows: A scaling approach, Croatica chemica acta 69, 793 (1996).

[32] O. V. Borisov, E. B. Zhulina, and T. M. Birshtein, Diagram of the states of a grafted polyelectrolyte layer, Macromolecules 27, 4795 (1994).

[33] S.-Z. He, H. Merlitz, L. Chen, J.-U. Sommer, and C.-X $\mathrm{Wu}$, Polyelectrolyte brushes: Md simulation and scf theory, Macromolecules 43, 7845 (2010).

[34] W. Ge, A. Encinas, E. Araujo, and S. Song, Magnetic matrices used in high gradient magnetic separation (hgms): A review, Res. Phys. 7, 4278 (2017).

[35] M. E. Cates and J. Tailleur, Motility-induced phase separation, Annu. Rev. Condens. Matter Phys. 6, 219 (2015).

[36] C. Bechinger, R. Di Leonardo, H. Löwen, C. Reichhardt, G. Volpe, and G. Volpe, Active particles in complex and crowded environments, Rev. Mod. Phys. 88, 045006 (2016).

[37] H. Kählert, J. Carstensen, M. Bonitz, H. Löwen, F. Greiner, and A. Piel, Magnetizing a Complex Plasma without a Magnetic Field, Phys. Rev. Lett. 109, 155003 (2012).

[38] H. Löwen, Active particles in noninertial frames: How to selfpropel on a carousel, Phys. Rev. E 99, 062608 (2019).

[39] L. M. C. Janssen, A. Kaiser, and H. Löwen, Aging and rejuvenation of active matter under topological constraints, Sci. Rep. 7, 5667 (2017).

[40] T. Speck and R. L. Jack, Ideal bulk pressure of active brownian particles, Phys. Rev. E 93, 062605 (2016).

[41] C. Gardiner, Stochastic methods, 4th ed. (Springer, Berlin, 2009), Chap. 4.

[42] P. McCord Morse, H. Feshbach et al., Methods of Theoretical Physics, Vol II (McGraw-Hill, New York, 1953), Chap. 1.

[43] H. C. Brinkman, Brownian motion in a field of force and the diffusion theory of chemical reactions, Physica 22, 29 (1956).

[44] J. Tailleur and M. E. Cates, Statistical Mechanics of Interacting Run-And-Tumble Bacteria, Phys. Rev. Lett. 100, 218103 (2008).

[45] A. Sharma and J. M. Brader, Communication: Green-Kubo approach to the average swim speed in active Brownian systems, J. Chem. Phys. 145, 161101 (2016).

[46] L. Van Hove, Correlations in space and time and born approximation scattering in systems of interacting particles, Phys. Rev. 95, 249 (1954).

[47] J.-P. Hansen and I. R. McDonald, Theory of Simple Liquids, 4th ed. (Elsevier, Amsterdam, 1990), Chap. 7.

[48] E. Jones, T. Oliphant, P. Peterson et al., SciPy: Open source scientific tools for PYTHON, (2001-). 